\title{
Écrire (d')après Proust : citations et influences proustiennes dans la deuxième partie du Jardin des Plantes de Claude Simon
}

\author{
Chiara Falangola \\ Université de Colombie-Britannique
}

Affirmer l'influence proustienne sur l'écriture de Claude Simon est presque devenu une évidence qui devrait, peut-être, rester implicite. Combien de fois les lecteurs simoniens ont relevé l'amour des longues phrases, l'usage des parenthèses, des combinaisons chromatiques ou celui d'un lexique proustien? Maintes fois Proust a été défini comme le père-littéraire de Simon, son «novelist-progenitor ${ }^{1}$ » (Orr, 1993, p. 106). Mary Orr a déjà analysé leur rapport en démontrant comment, avec

1 Je traduis : « le père-littéraire ». 
La Bataille de Pharsale (1969), Simon dépasse " the anxiety of influence ${ }^{2}$ » (Bloom, 1975) en acquérant une " [i]ndependence of style and form ${ }^{3}$ » (Orr, 1993, p. 136) tout en étant toujours, sans équivoque, sous l'influence proustienne. Selon Orr, c'est avec Les Géorgiques (1981) et L'Acacia (1989) que Simon se détache irrévocablement de son père littéraire à travers son propre «post-Proustian, use of association, intertextual and intratextual reverberation 4 » (p. 137).

Nous verrons comment, dans Le Jardin des Plantes (1997), Simon tire les leçons de sa fréquentation de l'œuvre de Proust et combine des fragments textuels pour construire la deuxième partie du roman comme un «bloc indivisible» (Simon, 1958, p. 5). Le Jardin des Plantes est un roman fragmentaire sur les plans typographique, thématique, temporel et spatial, un texte où des blocs narratifs, relatant différents épisodes de la vie du narrateur, se juxtaposent à des fragments-citations d'autres textes, dont, par exemple, Sodome et Gomorrhe de Proust, Their Finest Hour de Churchill et La Guerre sans haine de Rommel. Nous étudierons en particulier la deuxième partie du roman de Simon, celle où l'influence proustienne arrive jusqu'au cut-up de la visite de la marquise de Cambremer à Balbec, scène mondaine qui sert de toile de fond à un exemple mémorable du génie proustien de l'écriture du temps. Chez Proust, les mouettes sur la mer, que Marcel compare aux nymphéas de Monet, figurent le passage du temps depuis cinq heures de l'après-midi jusqu'au coucher du soleil : au fil des pages, le

\footnotetext{
2 Je traduis : « l’anxiété de l'influence ».

3 Je traduis : « indépendance formelle et stylistique ».

4 Je traduis : "emploi après-Proust de l'association et de la réverbération intertextuelle et intratextuelle».
} 
narrateur écrit les reflets de la couleur changeante du soleil et sa lumière répandue sur le plumage des mouettes.

Cet emprunt littéraire sera analysé textuellement et thématiquement: d'abord, nous suivrons l'intégration des fragments proustiens aux autres blocs narratifs, intégration qui se fait selon une logique compositionnelle du contraste; nous verrons ensuite comment la "conversation-Poussin» entre Marcel et la Marquise fournit à la construction romanesque une poétique de couchers de soleil; enfin, nous mettrons en lumière une certaine réflexion sur l'art romanesque dans laquelle se sont insérés Valéry, Breton et Proust avant d'en arriver à Simon.

\section{Une composition par contrastes}

Simon lui-même a commenté l'emprunt littéraire proustien pour éclairer le processus de création de la deuxième partie de son roman:

On fait comme on peut. On joue avec des alternances, des oppositions, des assonances, des dissonances, etc. Par exemple quand j'introduis au milieu d'un récit de guerre des citations de Proust, cette conversation paisible - et quelque peu comique - avec la marquise de Cambremer (que le lift s'obstine à appeler Camembert) sur la terrasse du Grand Hôtel, à Balbec, à la même heure (cinq heures de l'après-midi) où Rommel a lancé son offensive [...]. De plus encore, il y a parfois des chevauchements, des sortes d'échos. (1998)

Il s'agit d'une poétique du contraste qui exploite les effets de juxtaposition des blocs narratifs antinomiques. L'image matricielle du Jardin des Plantes, roman-labyrinthe construit sur des détours, des retours en arrière et des ellipses, est une image de guerre: l'épisode de la marche du cavalier $\mathrm{S}$. avec 
deux colonels. La clé interprétative de cet épisode sera livrée dans la troisième partie du roman lorsque $S$. le romancier est interviewé par un journaliste. Cette deuxième partie prépare la révélation qui suivra et encadre l'épisode de la cavalcade dans « un paradoxal et riant décor printanier» (Simon, 2006, p. 1025):

Les trajectoires respectivement suivies par $S$. et $R$. se trouveront confondues sur une courte distance (d'environ cinq kilomètres), chacune empruntant à quelques heures d'intervalle la route qui mène de Sorle-le-Château à Avesnes, [...] où l'un des deux colonels suivis par $S$. (qui, à ce moment, constituera avec un autre cavalier toute leur escorte) sera abattu. Il sera alors environ 10 ou 11 heures du matin. Il fera très beau et le soleil printanier brillera sur les prés, les haies d'aubépine en fleurs, les vergers, teintant de saumon les fumées qui çà et là s'élèveront parfois de véhicules achevant de se consumer, étincelant sur les toits d'ardoise des fermes. (Simon, 2006, p. 1017)

Le futur de narration utilisé dans ce bloc crée un effet de tension narrative en avant, vers la constitution d'une image, dont le moment final semble guetté par l'urgence d'une fatalité qui s'impose comme une nécessité, toutefois impénétrable. De fait, la clé interprétative de cet épisode réside dans l'opposition paradoxale entre le désir de vivre provoqué par l'évidence d'une nature vivante et printanière et le sentiment d'une mort imminente, perçue comme une condamnation arbitraire et inattendue. De ce contraste absurde entre l'urgence de la vie et l'apparente nécessité de la mort naît une sensation, et non pas un sentiment, que le narrateur définit comme "mélancolie ${ }^{5}$ ». Une mélancolie des sens et non pas du cœur : un attachement à

5 Cette sensation mélancolique fait penser au Meursault de la fin de L'Étranger : «Alors, je ne sais pas pourquoi, il y a quelque chose qui a crevé en moi. » (p. 119-20). 
la vie et à l'expérience de celle-ci, une sensation déclenchée par le contraste entre l'imminence de la mort et la vue paisible de la campagne printanière ${ }^{6}$. Dans «la tendre indifférence du monde » (Camus, 2004, p. 122), l'escadron du chevalier S. se trouve ainsi placé dans une embuscade due à la négligence des autorités militaires françaises. La condition humaine, dont le contexte spatio-temporel est totalement injustifié, possède un statut non nécessaire, hasardeux : la logique de la composition en textualise le tragique en filtrant le sens du paradoxe et de l'absurde dans ses images. Cependant, cette même logique compositionnelle lui impose une nécessité textuelle, comme nous le verrons plus loin. Le contraste antinomique part de cet épisode et reste présent sous forme de cercles concentriques jusqu'à la fin de la deuxième partie du roman. Le premier cercle autour de ce centre générateur correspond à l'entretien du romancier $\mathrm{S}$. avec le journaliste lors duquel les deux personnages se parlent sans se comprendre. Une série d'images antinomiques, pointant le paradoxe entre la vie et la mort, dérive de l'image matricielle de la marche lors de la mort du colonel et de ce dialogue de demi-sourds entre $S$. et le journaliste. Dans la deuxième partie du Jardin des Plantes, la logique compositionnelle du contraste opère soit par images antithétiques, soit par la juxtaposition des blocs narratifs antinomiques.

Je me contenterai de l'exemple des tout premiers blocs narratifs de cette section. Dans le premier, des piquets d'honneur, lors de la visite de la reine au Quai d'Orsay en juin

6 À propos de la mélancolie dans LeJardin des Plantes, voir aussi Houppermans (2001). 
1992, sont "comme un démenti joyeux, carnavalesque et bariolé aux tenues de combat, l'antithèse optimiste et pimpante de la boue des champs de bataille, des uniformes couleur de terre et de la souffrance » (p. 1015 ; je souligne). Le bloc narratif suivant, qui est un autre fragment du récit de la marche avec le colonel en mai 1940, s'ouvre sur un cratylisme topographique très proustien :

[L]a campagne [...] parsemée d'étangs, de villages dont les noms (Cerfontaine, Sivry, Clairfayts, Eppe-Sauvage) aux consonances d'eaux vives, de fraîcheur et d'ombrages semblaient contredire l'idée même de guerre, de mort, sauf ici et là, dans les prés [...], les cadavres de quelques vaches atteintes par un éclat perdu [...] leurs quatre pattes dressées vers le ciel comme des piquets dans une dernière et muette protestation » (p. 1016 ; je souligne).

Ce fragment est parfaitement soudé au précédent non seulement par la répétition du mot "piquets», mais aussi par l'homogénéité de l'imaginaire qui nourrit la comparaison de la première citation et la description de la seconde, ainsi que par la référence au contraste rhétorique des mots " antithèse » et « contredire». Tout comme le fragment du colonel mentionné plus haut, ces blocs narratifs sont construits sur la différence essentielle entre deux champs lexicaux et deux versants inconciliables de l'existence : la sérénité et la souffrance, la vie et la mort. En outre, la «muette protestation» des vaches anticipe la révélation sur la « mélancolie » de la troisième partie du Jardin et prépare le fragment de l'épisode de la cavalcade, qui arrive deux blocs narratifs plus tard.

En outre, le fragment relatif au souvenir de guerre du narrateur est suivi par le récit de la visite de Churchill au Quai d'Orsay, dont l'analyse sera repoussée à la deuxième partie de 
cette étude en tant que point de départ de son développement ultérieur. Successivement, le narrateur du Jardin des Plantes, qui se fait lecteur-critique de Proust, révèle l'astuce narrative proustienne du fameux passage des mouettes-nymphéas :

Quoique Proust ne donne pas d'indications précises, on peut déduire du contexte qu'il doit être environ 5 heures de l'aprèsmidi lorsque accompagnée de sa bru, née Legrandin, la marquise de Cambremer (que le groom de l'hôtel s'obstine à appeler Camembert) vient s'installer avec le narrateur et Albertine sur la terrasse du Grand Hôtel. « Nous regardions la mer calme où des mouettes éparses flottaient comme des corolles blanches », écrit Proust en effet, ce qui donne à penser (comme on pourra le voir au cours du récit où les mouettes changent peu à peu de couleur tandis que l'heure avance) qu'au moment où s'installent la marquise et ses amis la lumière (on est au mois de mai) ne se teinte pas encore de ces irisations qu'elle prend au fur et à mesure que le soleil décline : « [...] je me mis instinctivement à parler à Mme de Cambremer, née Legrandin, de la façon qu'eût pu faire son frère. "Elles ont, disje, en parlant des mouettes, une immobilité et une blancheur de nymphéas." ». (p. 1018)

À première lecture, cette citation-commentaire de Proust semble se lier par opposition au fragment suivant, en jouant sur la dissonance entre la prose proustienne et la désolation de l'image qui suit. En fait, une lecture plus attentive manifeste une homogénéité fondamentale: "La scène suivante se déroule environ vingt ans après l'épisode de la mort du colonel » (p. 1019). Ici, le mot "scène» et le verbe «dérouler» sensibilisent le lecteur à la mobilité, à un dynamisme auquel on s'attend. Le texte engage visuellement le lecteur qui, attentif aux détails, observe "une jeune fille (ou une très jeune femme) vêtue de rose [qui] sort de la station métro Stadtmitte (Centreville) à Berlin. Celle-ci s'ouvre au milieu d'une vaste étendue de ruines [...]. » (p. 1019) S'en suit une assez longue description de 
la place et de ses ruines. La pause descriptive met le lecteur en attente jusqu'au moment où, une vingtaine de lignes plus bas, réapparaît sur scène cette jeune fille en rose:

La jeune fille en robe rose qui sort de la station de métro est vêtue avec élégance, comme pour quelque rendez-vous ou pour se rendre à quelque fête. D'une démarche gracieuse, elle se dirige parmi les entassements de décombres vers ce qui n'est plus maintenant qu'une trouée, bordée d'un côté de vagues tas de briques et, de l'autre, d'une suite de façades restées debout, sans rien derrière, comme un décor de théâtre aux fenêtres vides à travers lesquelles on peut voir le ciel. Le silence n'est parfois troublé que par les bruits lointains de la circulation dans la partie reconstruite de la ville, comme un faible chuintement, pas plus fort que celui dans lequel se déroule un film muet. [...] La silhouette de la fille en rose qui en longe la base diminue peu à peu, sa jupe se balançant au rythme gracieux de ses pas. Bientôt elle n'est plus qu'une tache dansante qui diminue encore, un point, puis plus rien, disparue. (p. 1020 ; je souligne)

La jeunesse, la couleur rose et la légèreté de la jeune fille contrastent avec les ruines de guerre: malgré tout, la vie continue et s'épanouit. Non seulement ce bloc se construit comme les autres sur le contraste fondamental entre la vie et la mort, mais la couleur rose, la grâce et l'ondulation circulaire de la jupe, l'anti-climax visuel constitué par la succession des mots "silhouette», "tache» et "point», ne peuvent pas ne pas rappeler les nymphéas de Monet et instaurer une homogénéité avec la citation proustienne. La jeune fille est une tache de couleur qui se balance parmi les ruines de Berlin, qui semble ondoyer légèrement comme un nymphéa sur l'eau.

Cette construction descriptive de la fille en rose est une véritable image-carrefour qui non seulement participe de la poétique du contraste paradoxal et de l'intertexte proustiens, 
mais s'ajoute en troisième terme au binôme mouettesnymphéas et annonce enfin l'image suivante, celle d'un coucher du soleil qui disparaît progressivement derrière les ruines.

\section{Une construction chromatique}

Revenons au bloc narratif du récit de la visite de Churchill au Quai d'Orsay, en 1940, à la suite de l'attaque de Rommel sur la Meuse. Comme nous l'avons déjà dit, le fragment sur Churchill suit celui de la mort du colonel, image centrale de la deuxième partie du roman: «Il fera très beau et le soleil printanier brillera sur les prés, les haies d'aubépine en fleurs, les vergers teintant de saumon les fumées qui çà et là s'élèveront parfois de véhicules achevant de se consumer, étincelant sur les toits d'ardoise des fermes.» (p. 1017). Les véhicules incendiés servent de pont textuel avec le bloc narratif consacré à Churchill, dont l'image capitale est celle d'un autre incendie. Le premier ministre anglais arrive au Quai d'Orsay dans «le gai soleil de mai obscurci par les noires volutes de fumée qu'avec tout juste un froncement étonné de sourcils il fit semblant de ne pas voir » (p. 1017-8). Il est cinq heures trente de l'après-midi, le 16 mai 1940. Les jours précédents, les deuxième et neuvième armées françaises sont tombées dans une embuscade et les Allemands sont arrivés sur le territoire français. La fumée qui monte des jardins du ministère des Affaires étrangères est celle des archives brûlées par des employés du ministère à la suite de la débâcle de l'armée. À partir du bloc narratif sur l'incendie de cinq heures de l'après-midi, Simon développe une suite presque obsessionnelle d'allusions et d'images de feux et de couchers de soleil, construite sur le calque de l'intertexte proustien, c'est-à-dire de façon chromatique et à travers la 
référence à " cinq heures de l'après-midi », heure à laquelle la marquise de Cambremer, sa bru née Legrandin, Marcel et Albertine s'installent sur la terrasse du Grand Hôtel de Balbec. Après le bloc narratif de la fille en rose, dans lequel deux églises baroques «touchées par les bombes et incendiées, sont maintenant d'un rouge noirci, couleur de briques cuites », un fragment de la citation proustienne se référant au chromatisme d'un coucher de soleil apparaît ensuite : «il était malheureux qu'elle n'eût pas eu plutôt l'idée de venir la veille, car à la même heure, c'est une lumière de Poussin qu'elle eût pu admirer » (p. 1020). Dans le bloc narratif qui suit, à 5 heures de l'aprèsmidi, au Quai d'Orsay, Simon nous livre un fragment de la visite de la reine d'Angleterre, où une femme en rouge, "une photographie en couleurs représentant la cheminée d'un des salons du palais où se tient la réception » (p. 1021), la flamme d'une torche et le soleil créent un effet textuel dont la dominante chromatique rappelle une lumière de Poussin (voir Ferrato-Combe). Ensuite, s'inscrivent, dans cette série-Poussin, une photographie de Churchill au coucher de soleil peignant des couchers de soleil (voir p. 1024) et, à nouveau, la marquise de Cambremer s'exclamant: "J'ai horreur des couchers de soleil, c'est romantique, c'est opéra.” » (p. 1024). Cette série de couchers de soleil, où le narrateur scande l'heure dans presque chaque bloc narratif, crée un effet polyphonique tendu autour de cet intervalle temporel de 5 à 6 heures. Dans les fragments de la visite de Churchill et de la réception de la reine Élisabeth au Quai d'Orsay, l'œil descripteur est hanté par la même pendule et le même cadran d'horloge:

Les mêmes chérubins de stuc aux nudités voilées d'or, avec leurs mêmes fossettes, leurs mêmes visages mutins, répétés de part et d'autre de la cheminée, au-dessus des frontons, des 
chapiteaux de stuc, la même nymphe d'albâtre drapée d'albâtre, la même pendule au cadran d'émail, aux chiffres romains (I, II, III, IV, V, VI... X, XII..., comme si le temps, l'Histoire, devaient toujours être jalonnés par ces barres, ces signes géométriques, ces coins, ces croix gravées par des Césars), les aiguilles de bronze doré minutieusement ouvragées indiquant 5 heures et demie de l'après-midi lorsque tout juste débarqué de l'avion qui l'a amené de Londres [Churchill] fait son entrée. (p. 1023)

Comme Proust, Simon a sa manière d'évoquer le temps, manière qui dévoile sa vision du monde. $\mathrm{Si}$, chez Proust, le temps est destructeur et porteur des changements, chez Simon, il est plutôt question d'un temps répétitif, monotone et circulaire, ce qui nie toute idée de progrès à l'Histoire et affirme le retour du même, dans une sorte de simultanéisme narratif.

Vers la fin de la deuxième partie du Jardin des Plantes, dans un des fragments de l'interview de S., le dialogue se fait autoréflexif. S. demande : "Mais comment imaginer tout ça?...» (p. 1055) et le journaliste lui répond: «L'ensemble? Vous voulez dire se faire une idée de tout ce qui se passait au même moment? [...] Comme on a essayé de le faire au cinéma, il faudrait plusieurs écrans sur lesquels on projetterait simultanément des images différentes. » (p. 1055). C'est exactement ce que Simon fait dans la deuxième partie du Jardin des Plantes.

\section{La nécessité textuelle}

Pour comprendre les enjeux d'une telle pratique textuelle visant à un effet de simultanéité, nous nous pencherons sur les enjeux entre l'exergue de la deuxième partie du Jardin, les circonstances de la scène proustienne et sur la réminiscence littéraire de «[I]a marquise sortit à cinq heures » (Breton, 1962, p. 19). 
La deuxième partie du Jardin des Plantes s'ouvre sur cette citation : «On a recensé 367 démonstrations différentes du théorème de Pythagore.» (2006, p. 1013) En 1991, au cours d'un entretien, Simon affirmait : "Mais l'autre jour, la simple démonstration du théorème de Pythagore m'a comblé d'une jouissance infiniment plus grande (on touche là à une parfaite perfection de l'esprit) qu'un poème de Baudelaire. » (1991, p. 21). La jouissance dont parle Simon est de type esthétique et réside dans la beauté synthétique de l'expression mathématique d'un concept. Dans le langage de tous les jours, il y a toujours un écart dans la communication, dû à la différence des contextes énonciatifs des locuteurs, comme c'est le cas lorsque S. parle avec le journaliste dans Le Jardin. Mais dans un énoncé mathématique, il n'y a qu'un sens dépourvu d'équivoque et on a l'impression que le langage reflète, pour une fois, la vérité dans une volonté d'assertion élégante et synthétique. La démonstration d'une proposition permet d'en attester la vérité : démontrer veut dire fonder une certitude de vérité sans exception, c'est-à-dire lui conférer un pouvoir de nécessité. À la suite de l'enchaînement logique de plusieurs vérités nécessaires, on arrive à une vérité exacte et universelle. L'exergue scientifique nous semble faire contrepoint avec le souci fondamental de Simon - l'arbitraire de l'Histoire - , avec la contingence de la condition historique de l'homme et, enfin, avec la contingence romanesque, marquée par la Marquise de Cambremer et l'insistance faite aux cinq heures de l'après-midi. En effet, dans le Manifeste du surréalisme, Breton, s'attaquant aux romans dits réalistes, rappelle le célèbre La marquise sortit à cinq heures, formule qui, pour Valéry, représentait « [l]e caractère circonstanciel, inutilement particulier» (Breton, 1962, p.19) du genre romanesque. Cette formule figure le 
paradigme de la contingence romanesque, de l'arbitraire et de l'interchangeabilité de son contenu. L'exergue mathématique évoque un discours qui relève au contraire de l'ordre de la nécessité, un discours où la causalité et l'enchaînement logique seraient incontournables. Par-delà l'arbitraire absurde inscrit dans les thématiques du Jardin des Plantes (la débâcle de l'armée, le manque de coordination des opérations militaires, la mort du colonel, l'Histoire en soi, etc.), cet exergue indique la possibilité d'une construction nécessaire.

« Mais comment imaginer tout ça? » et donner « une idée de tout ce qui se passait au même moment»? Simon relève le défi posé à la fois par son personnage ainsi que par Valéry et postule une sorte de nécessité textuelle qui se passe de la chronologie et de la progression causale du roman traditionnel. Il s'ingénie à créer un effet de simultanéité et insiste sur la synchronicité textuelle des scènes appartenant à différents fragments narratifs. Les blocs narratifs se lient ensemble en fonction non de relations causales, mais de la nature des images, des couleurs ou grâce à la matérialité du signifiant de certains mots.

La simultanéité à laquelle vise l'arrangement textuel suggère un schéma compositionnel qui n'est pas circonstanciel : la poétique du contraste, les couleurs de l'incendie des archives et des couchers de soleil proustiens, la synchronicité des événements relatés imposent un ordre de composition textuelle nécessaire.

Le Jardin des Plantes figure parmi les romans de la maturité littéraire simonienne (d'après 1969, selon Orr), parmi ceux qui, 
par leur structure et leur logique, utilisent la citation, l'allusion ou l'intertexte pour en faire des éléments tout à fait originaux et attestent de la pleine émancipation de l'écrivain vis-à-vis du modèle littéraire traditionnel. L'épisode de la marquise de Cambremer à Balbec et des mouettes-nymphéas, que Simon emprunte à Proust, non seulement remplit une fonction de contraste avec le récit de la débâcle de l'armée française en maijuin 1940, mais décide des couleurs dominantes de la deuxième partie du Jardin des Plantes.

Simon rend hommage à Proust tout en poursuivant sa propre exploration des possibles du roman. Il inscrit le sens d'un tel intertexte dans la structure antinarrative de son univers romanesque. D’un côté, il construit son propre récit d'une façon chromatique; de l'autre, il remplace la chronologie et la causalité narrative par une tentative de juxtaposition immédiate des événements racontés. C'est à travers le difficile travail textuel de composition et recomposition de l'expérience de la guerre que Simon à la fois rend hommage à Proust tout en s'en distinguant. L'écriture simonienne ( $d$ ')après Proust, qui est en même temps post-proustienne et selon Proust, serait alors moins à chercher dans les citations, dans la référence au temps ou aux jeux de couleurs, que dans le fait d'avoir appris la véritable leçon de la Recherche :

[L]e style pour l'écrivain aussi bien que la couleur pour le peintre est une question non de technique mais de vision. Il est la révélation, qui serait impossible par des moyens directs et conscients, de la différence qualitative qu'il y a dans la façon dont nous apparaît le monde, différence qui, s'il n'y avait pas l'art, resterait le secret éternel de chacun. (Proust, 2008, p. 202) 


\section{Bibliographie}

BLoom, Harold. (1975), The Anxiety of Influence, Oxford, University Press.

BRETON, André. (1962 [1924]), Manifestes du surréalisme, Montreuil, Jean-Jacques Pauvert.

CAmus, Albert. (1996 [1942]), L'Étranger, Paris, Gallimard.

FERrato-Combe, Brigitte. (2001), «Dans une lumière de Poussin... », dans S. Houppermans (dir.), Claude Simon et Le Jardin des Plantes, Amsterdam, CRIN 39, p. 119-131.

HoupPeRmANS, Sjef. (2001), «Claude Simon et Samuel Beckett :

Pour la mémoire du siècle », dans S. Houppermans (dir.), Claude Simon et Le Jardin des Plantes, Amsterdam, CRIN 39, p. 133-145.

ORR, Mary. (1993), Claude Simon: The Intertextual Dimension, University of Glasgow French and German Publications.

Proust, Marcel. (2008 [1927]), Le Temps retrouvé, Paris, Gallimard.

Simon, Claude. (2006), OEuvres, Paris, Gallimard, coll. «Bibliothèque de la Pléiade ».

—. (1958), "“Un bloc indivisible”, réponse à la question : "Pourquoi des romans?" », Les Lettres françaises no 750, 4-10 décembre, p. 5.

—. (1991), "... peuvent et doivent parfois s'arrêter d'écrire", entretien avec Bernard-Henri Lévy, dans B.-H. Lévy, Les Aventures de la liberté: une histoire subjective des intellectuels, Paris, Grasset, p. 12-21. 
-. (1998), "Parvenir peu à peu à écrire difficilement», entretien avec Jean-Claude Lebrun, L'Humanité, 13 mars [http://www.humanite.fr/node/332167], cité dans C. Simon, CEuvres, 2006, note 6 de la deuxième partie du Jardin des Plantes par Jean H. Duffy et Alastair B. Duncan, p. 1510.

\title{
Résumé
}

Dans la deuxième partie du Jardin des Plantes de Claude Simon, l'influence proustienne arrive jusqu'au cut-up de la visite de la marquise de Cambremer à Balbec dans Sodome et Gomorrhe. Les mouettes-nymphéas que Simon emprunte à Proust non seulement remplissent une fonction de contraste avec le récit de la débâcle de l'armée française en mai/juin 1940, mais décident des couleurs dominantes de cette deuxième partie du Jardin des Plantes. En jouant textuellement avec le passage du blanc au rose des mouettes, qui figuraient chez Proust le passage du temps dans les couleurs du coucher de soleil, Simon construit son propre récit fragmentaire et hétérogène - d'une façon chromatique, selon une nouvelle nécessité textuelle.

\begin{abstract}
In the second part of The Jardin des Plantes, a novel by Claude Simon, Proust's influence reaches the cut-up of the Marquise's de Cambremer episode in Sodom and Gomorrah. The seagullswaterlilys that Simon borrows from Proust serve two purposes: to establish a contrast with the 1940 Battle of France and to set the main colors of the chapter. Simon builds his fragmentary and heterogeneous novel in a chromatic way, by playing with the shift in colors from white to pink of the seagulls. In Proust, the latter represented the passing of time through the changing colors of the sunset; in Simon, a color arrangement is created according to a new textual necessity.
\end{abstract}

